\title{
Some combinatorial identities for the $r$-Dowling polynomials
}

\author{
Mark Shattuck \\ Institute for Computational Science \& Faculty of Mathematics and Statistics \\ Ton Duc Thang University, Ho Chi Minh City, Vietnam \\ e-mail: mark. shattucketdtu.edu.vn
}

Received: 12 March 2019

Accepted: 8 April 2019

\begin{abstract}
Recently, three new Bell number formulas were proven using algebraic methods, one of which extended an earlier identity of Gould-Quaintance and another a previous identity of Spivey. Here, making use of combinatorial arguments to establish our results, we find generalizations of these formulas in terms of the $r$-Dowling polynomials. In two cases, weights of the form $a^{i}$ and $b^{j}$ may be replaced by arbitrary sequences of variables $x_{i}$ and $y_{j}$ which yields further generalizations. Finally, a second extension of one of the formulas is found that involves generalized Stirling polynomials and leads to analogues of this formula for other counting sequences.
\end{abstract}

Keywords: Bell numbers, $r$-Dowling polynomials, $r$-Whitney numbers, Polynomial generalization.

2010 Mathematics Subject Classification: 05A19, $11 B 73$.

\section{Introduction}

Given integers $r \geq 0$ and $m \geq 1$, let $W_{m, r}(n, k)$ denote the $r$-Whitney numbers of the second kind which are determined by the polynomial identities

$$
(m x+r)^{n}=\sum_{k=0}^{n} W_{m, r}(n, k) m^{k} x^{\underline{k}}, \quad n \geq 0,
$$

where $x^{\underline{k}}=x(x-1) \cdots(x-k+1)$. The $W_{m, r}(n, k)$ were introduced in [8] and later considered in [2] in conjunction with a certain kind of finite geometric lattice known as the Dowling 
lattice [3]. The $r$-Whitney numbers satisfy the recurrence

$$
W_{m, r}(n, k)=W_{m, r}(n-1, k-1)+(r+m k) W_{m, r}(n-1, k), \quad n, k \geq 1,
$$

with $W_{m, r}(n, 0)=r^{n}$ and $W_{m, r}(0, k)=\delta_{k, 0}$ for all $n, k \geq 0$. Note that $W_{1,0}(n, k)=\left\{\begin{array}{l}n \\ k\end{array}\right\}$, where $\left\{\begin{array}{l}n \\ k\end{array}\right\}$ denotes the classical Stirling number of the second kind (see [13, Sequence A008277]).

The $r$-Dowling polynomials, which we will denote here by $D_{m, r}(n ; y)$, are defined as

$$
D_{m, r}(n ; y)=\sum_{k=0}^{n} W_{m, r}(n, k) y^{k}, \quad n \geq 0,
$$

where $y$ is an indeterminate. In [2], some properties of $D_{m, r}(n ; y)$ were found using Riordan matrices. See also $[1,11]$, where the case $r=1$ is studied. Note that the $D_{m, r}(n ; y)$ reduce when $r=0$ and $m=y=1$ to the classical Bell numbers $B(n)$, see [13, A000110].

A partition of $[n]=\{1,2, \ldots, n\}$ is a collection of non-empty, mutually disjoint subsets, called blocks, whose union is $[n]$. Recall that $\left\{\begin{array}{l}n \\ k\end{array}\right\}$ gives the number of partitions of $[n]$ having exactly $k$ blocks, while $B(n)$ is the total number of partitions of $[n]$. Let $\left[\begin{array}{l}n \\ k\end{array}\right]$ denote the (signless) Stirling number of the first kind which gives the number of permutations of $[n]$ having $k$ cycles. The following Bell number formulas where $n$ and $k$ are non-negative integers were shown recently by Komatsu and Pita-Ruiz [5] using algebraic methods:

$$
\begin{gathered}
\sum_{j=0}^{n}\left(\begin{array}{l}
n \\
j
\end{array}\right) B(k+j)=\sum_{j=0}^{k}\left(\begin{array}{l}
k \\
j
\end{array}\right)(-1)^{k-j} B(n+j+1), \\
\sum_{j=0}^{n}\left(\begin{array}{l}
n \\
j
\end{array}\right) a^{j} b^{n-j} B(j)=\sum_{j=0}^{k}(-1)^{k-j}\left[\begin{array}{c}
k \\
j
\end{array}\right] \sum_{\ell=0}^{n}\left(\begin{array}{l}
n \\
\ell
\end{array}\right) a^{\ell}(b-a k)^{n-\ell} B(\ell+j),
\end{gathered}
$$

and

$$
\sum_{j=0}^{n+k}\left(\begin{array}{c}
n+k \\
j
\end{array}\right) a^{j} b^{n+k-j} B(j)=\sum_{p=0}^{k} \sum_{\ell=0}^{k} \sum_{j=0}^{n} \sum_{i=0}^{j}\left(\begin{array}{l}
n \\
j
\end{array}\right)\left(\begin{array}{l}
k \\
\ell
\end{array}\right)\left(\begin{array}{l}
j \\
i
\end{array}\right) a^{n+\ell+i-j} b^{k+j-\ell-i} p^{n-j}\left\{\begin{array}{l}
\ell \\
p
\end{array}\right\} B(i)
$$

In (4) and (5), the $a$ and $b$ represent arbitrary complex numbers (or indeterminates). Identities (3)-(5) may be rewritten in recurrent form by isolating the term containing $B(q)$ with the greatest $q$, a feature that will also be shared by the generalizations below.

Note that (4) reduces when $a=1$ and $b=0$ to

$$
B(n)=\sum_{j=0}^{k}(-1)^{k-j}\left[\begin{array}{l}
k \\
j
\end{array}\right] \sum_{\ell=0}^{n}\left(\begin{array}{l}
n \\
\ell
\end{array}\right)(-k)^{n-\ell} B(\ell+j), \quad n, k \geq 0
$$

which was originally shown by Gould and Quaintance [4, Theorem 2], while (5) for the same values of $a$ and $b$ reduces to

$$
B(n+k)=\sum_{p=0}^{k} \sum_{j=0}^{n}\left(\begin{array}{l}
n \\
j
\end{array}\right) p^{n-j}\left\{\begin{array}{l}
k \\
p
\end{array}\right\} B(j), \quad n, k \geq 0
$$

which was shown by Spivey [14, Formula 3]. See also [9, 12, 15] for other extensions of (7). 
In this paper, we provide generalizations of (3)-(5) above in terms of the $r$-Dowling polynomials $D_{m, r}(n ; y)$ which will reduce to these formulas when $m=y=1$ and $r=0$. See Theorems 2.3, 2.4 and 2.5 below. We make use of combinatorial proofs to establish our results which specialize to provide new proofs of (3)-(5). As a consequence of our arguments, we are able to replace the factors of $a^{j}$ and $b^{j}$ appearing in formulas (4) and (5) with arbitrary sequences $x_{j}$ and $y_{j}$. We also consider a further extension of (5) in terms of generalized Stirling polynomials, which will yield analogues of (5) for other types of counting sequences.

\section{$2 r$-Dowling generalizations of identities (3)-(5)}

In this section, we present generalizations of identities (3)-(5) involving the $r$-Dowling polynomials. We first describe a combinatorial interpretation for $W_{m, r}(n, k)$ and $D_{m, r}(n ; y)$ from [6] which we will make use of in the subsequent proofs.

Definition 2.1. If $0 \leq r \leq s$, then an $r$-partition of $[s]$ is a partition of the elements of $[s]$ in which the elements of $[r]$ belong to distinct blocks. If $n, k, r \geq 0$, then let $\mathcal{B}_{r}(n, k)$ denote the set of r-partitions of $[n+r]$ having $k+r$ blocks and let $\mathcal{B}_{r}(n)=\cup_{k=0}^{n} \mathcal{B}_{r}(n, k)$.

Note that when $r=0$, the set $\mathcal{B}_{r}(n)$ is the same as the set of ordinary partitions of $[n]$. Within a member of $\mathcal{B}_{r}(n, k)$, a block containing an element of $[r]$ will be referred to as special, while the remaining $k$ blocks composed exclusively of elements of $[r+1, r+n]=\{r+1, \ldots, r+n\}$ are non-special. The members of $[r]$ themselves will also at times be described as special.

Definition 2.2. If $m$ is a positive integer, then let $\mathcal{B}_{m, r}(n, k)$ denote the set of $r$-partitions of $[n+r]$ having $k+r$ blocks such that within each non-special block, every element except the smallest is assigned one of $m$ colors, and let $\mathcal{B}_{m, r}(n)=\cup_{k=0}^{n} \mathcal{B}_{m, r}(n, k)$.

Upon making a comparison of the recurrences and initial values, one has $\left|\mathcal{B}_{m, r}(n, k)\right|=$ $W_{m, r}(n, k)$ for all relevant values of the parameters. Weighting members of $\mathcal{B}_{m, r}(n, k)$ by $y^{k}$ for $0 \leq k \leq n$, it is seen then from the definition that $D_{m, r}(n ; y)$ gives the sum of the weights of all members of $\mathcal{B}_{m, r}(n)$. (Alternatively, one may assume $y$ is a positive integer and that each non-special block within a member of $\mathcal{B}_{m, r}(n)$ is assigned one of $y$ colors.)

We first extend formula (3) above to $D_{m, r}(n ; y)$ in two different ways.

Theorem 2.3. If $n, k, r \geq 0$, then

$$
y \sum_{j=0}^{n}\left(\begin{array}{l}
n \\
j
\end{array}\right) m^{n-j} D_{m, r}(k+j ; y)=\sum_{j=0}^{k}\left(\begin{array}{l}
k \\
j
\end{array}\right)(-m)^{k-j}\left(D_{m, r}(n+j+1 ; y)-r D_{m, r}(n+j ; y)\right)
$$

and

$$
\sum_{j=0}^{n}\left(\begin{array}{l}
n \\
j
\end{array}\right) D_{m, r}(k+j ; y)=\sum_{j=0}^{k}\left(\begin{array}{l}
k \\
j
\end{array}\right)(-1)^{k-j} D_{m, r+1}(n+j ; y) .
$$


Proof. Considering whether or not the element $n+j+r+1$ within $\lambda \in \mathcal{B}_{m, r}(n+j+1)$ belongs to a special block and, if not, the cardinality $i+1$ of the block within which it lies, implies

$$
D_{m, r}(n+j+1 ; y)=r D_{m, r}(n+j ; y)+y \sum_{i=0}^{n+j}\left(\begin{array}{c}
n+j \\
i
\end{array}\right) m^{i} D_{m, r}(n-i+j ; y) .
$$

Thus, to establish (8), it suffices to show

$$
\sum_{t=0}^{n}\left(\begin{array}{l}
n \\
t
\end{array}\right) m^{n-t} D_{m, r}(k+t ; y)=\sum_{j=0}^{k}\left(\begin{array}{l}
k \\
j
\end{array}\right)(-m)^{k-j} \sum_{i=0}^{n+j}\left(\begin{array}{c}
n+j \\
i
\end{array}\right) m^{i} D_{m, r}(n-i+j ; y) .
$$

To do so, let $\mathcal{P}_{n, k, r}^{(j)}$ denote the set of ordered pairs $(\alpha, \beta)$, where $\alpha \subseteq[r+1, r+k]$ with $|\alpha|=$ $k-j$ and members of $\alpha$ are each colored in one of $m$ ways and $\beta$ is a partition of the set $[n+k+r+1]-\alpha$ where the elements of this set are arranged according to a member of $\mathcal{B}_{m, r}(n+j+1)$ in which $n+j+r+1$ belongs to a non-special block. Considering the cardinality $i+1$ where $0 \leq i \leq n+j$ of the block containing the largest element in $\beta$ then implies that there are $y \sum_{i=0}^{n+j}\left(\begin{array}{c}n+j \\ i\end{array}\right) m^{i} D_{m, r}(n-i+j ; y)$ possible $\beta$ for each $\alpha$. Let $\mathcal{P}_{n, k, r}=\cup_{j=0}^{k} \mathcal{P}_{n, k, r}^{(j)}$ and members of $\mathcal{P}_{n, k, r}^{(j)}$ have sign $(-1)^{k-j}$. Then the right-hand side of (10) multiplied by $y$ gives the sum of the signs of all members of $\mathcal{P}_{n, k, r}$.

If $(\alpha, \beta) \in \mathcal{P}_{n, k, r}$, then let $\ell^{*}$ be the smallest $\ell \in[r+1, r+k]$ (if it exists) such that either (i) $\ell \in \alpha$ or (ii) $\ell \notin \alpha$, with $\ell$ belonging to the block of $\beta$ containing $n+k+r+1$. Then the operation of switching options (i) and (ii) by moving $\ell^{*}$ from $\alpha$ to $\beta$ or vice versa (keeping its color the same in either case) is a sign-changing involution where it is defined. This operation is not defined for members of $\mathcal{P}_{n, k, r}^{*} \subseteq \mathcal{P}_{n, k, r}$ comprising those $(\alpha, \beta)$ where $\alpha=\varnothing$ (i.e., $j=k$ ) and the block of $\beta$ containing $n+k+r+1$ has no elements of $[r+1, r+k]$ in it. Then members $(\alpha, \beta)$ of $\mathcal{P}_{n, k, r}^{*}$ all have positive sign and have cardinality given by $y \sum_{t=0}^{n}\left(\begin{array}{c}n \\ n-t\end{array}\right) m^{n-t} D_{m, r}(k+t ; y)$, upon considering the number $n-t$ of additional elements in the block of $\beta$ containing $n+k+r+1$ (note that all of these elements must belong to $[r+k+1, r+k+n]$ ). Equating this with the previous expression for the sum of the signs of all members of $\mathcal{P}_{n, k, r}$ gives (10) and thus (8).

To show (9), first note that

$$
D_{m, r+1}(n+j ; y)=\sum_{i=0}^{n+j}\left(\begin{array}{c}
n+j \\
i
\end{array}\right) D_{m, r}(n-i+j ; y), \quad r \geq 0,
$$

upon considering the number $i$ of additional elements in the special block containing 1 within a member of $\mathcal{B}_{m, r+1}(n+j)$. Thus, to complete the proof of (9), it suffices to show

$$
\sum_{t=0}^{n}\left(\begin{array}{l}
n \\
t
\end{array}\right) D_{m, r}(k+t ; y)=\sum_{j=0}^{k}\left(\begin{array}{c}
k \\
j
\end{array}\right)(-1)^{k-j} \sum_{i=0}^{n+j}\left(\begin{array}{c}
n+j \\
i
\end{array}\right) D_{m, r}(n-i+j ; y) .
$$

For (11), we proceed in a similar manner as before and let $\mathcal{Q}_{n, k, r}^{(j)}$ denote the set of ordered pairs $(\gamma, \delta)$, where $\gamma \subseteq[r+2, r+k+1]$ with $|\gamma|=k-j$ and $\delta$ is a partition of the elements of $[n+k+r+2]-\delta$ arranged according to a member of $\mathcal{B}_{m, r+1}(n+j+1)$ in which $n+j+r+2$ belongs to the block containing 1 . Considering the number $i$ of additional non-special elements 
in the block containing 1 implies that there are $\sum_{i=0}^{n+j}\left(\begin{array}{c}n+j \\ i\end{array}\right) D_{m, r}(n-i+j ; y)$ possible $\delta$ for each $\gamma$. If the sign of $(\gamma, \delta)$ is defined as $(-1)^{|\gamma|}$, then the right side of (11) gives the sum of the signs of all members of $\mathcal{Q}_{n, k, r}=\cup_{j=0}^{k} \mathcal{Q}_{n, k, r}^{(j)}$.

We define an involution on $\mathcal{Q}_{n, k, r}$ by considering the smallest $\ell \in[r+2, r+k+1]$ that either belongs to $\gamma$ or to the block containing 1 within $\delta$, and switching to the other option. This operation reverses the sign and is not defined for those $(\gamma, \delta)$ such that $\gamma=\varnothing$ and the special block of $\delta$ to which 1 belongs contains $n+k+r+2$ but no elements of $[r+2, r+k+1]$. The cardinality of such $(\gamma, \delta)$ is given by the left side of (11), which completes the proof of (9).

If $n \geq 0$, then let $B(n ; y)=\sum_{k=0}^{n}\left\{\begin{array}{l}n \\ k\end{array}\right\} y^{k}$ denote the $n$-th Bell polynomial (see, e.g., [10]). Note $B(n ; y)=D_{1,0}(n ; y)$ and $B(n+1 ; y)=y D_{1,1}(n ; y)$. Taking $m=1$ and $r=0$ in both (8) and (9) yields the following identity for $B(n ; y)$.

Corollary 2.3.1. We have

$$
y \sum_{j=0}^{n}\left(\begin{array}{l}
n \\
j
\end{array}\right) B(k+j ; y)=\sum_{j=0}^{k}\left(\begin{array}{l}
k \\
j
\end{array}\right)(-1)^{k-j} B(n+j+1 ; y) .
$$

Note that (3) above corresponds to the $y=1$ case of (12). We now present a generalization of formula (4).

Theorem 2.4. If $n, k, r \geq 0$, then

$$
\begin{array}{r}
\sum_{j=0}^{n}\left(\begin{array}{l}
n \\
j
\end{array}\right) x_{j} y_{n-j} \sum_{i=0}^{r}\left(\begin{array}{l}
r \\
i
\end{array}\right)\left(\begin{array}{l}
k \\
i
\end{array}\right) i ! y^{k-i} D_{1, r-i}(j ; y)=\sum_{j=0}^{k}(-1)^{k-j}\left[\begin{array}{l}
k \\
j
\end{array}\right] \sum_{\ell=0}^{n} \sum_{i=0}^{\ell}\left(\begin{array}{l}
n \\
\ell
\end{array}\right)\left(\begin{array}{l}
\ell \\
i
\end{array}\right)(-k)^{\ell-i} \\
\times x_{n-i} y_{i} D_{1, r}(n-\ell+j ; y) .
\end{array}
$$

Proof. We will assume in the proof that $n, k, r \geq 1$, as the adjustments required when one of the parameters is zero will be apparent. We first describe a signed set of configurations for which the right-hand side of (13) is the sum of the signs. Given $n, k$ and $r$ with $0 \leq j \leq k, 0 \leq \ell \leq n$ and $0 \leq i \leq \ell$, let $\mathcal{P}_{n, k, r}^{(j, \ell, i)}$ denote the set of 4-tuples $(\alpha, \beta, \gamma, \delta)$ such that $\alpha$ is a permutation of $[k]$ containing $j$ cycles, $\beta$ is a subset of $[r+1, r+n]$ of size $n-\ell, \gamma$ is a subset of $[r+1, r+n]-\beta$ of size $\ell-i$ wherein each element of $\gamma$ is associated with an element contained within some cycle of $\alpha$, and $\delta$ is a partition of the cycles of $\alpha$ and the elements of $[r] \cup \beta$ arranged according to some member of $\mathcal{B}_{1, r}(n-\ell+j)$. It is assumed that cycles of $\alpha$ are written with the smallest element first in each cycle. We will refer to the elements of $\beta$ and $\gamma$ as free and associated, respectively. Let the sign of $\rho=(\alpha, \beta, \gamma, \delta) \in \mathcal{P}_{n, k, r}^{(j, \ell, i)}$ be given by $(-1)^{k-j+\ell-i}$ and the weight by $x_{n-i} y_{i} \operatorname{wght}(\delta)$, where $\operatorname{wght}(\delta)$ is the weight $\delta$ would receive if counted in $D_{1, r}(n-\ell+j ; y)$. Then the (signed) weight of all members of $\mathcal{P}_{n, k, r}^{(j, \ell, i)}$ equals

$$
(-1)^{k-j}\left[\begin{array}{l}
k \\
j
\end{array}\right]\left(\begin{array}{l}
n \\
\ell
\end{array}\right)\left(\begin{array}{l}
\ell \\
i
\end{array}\right)(-k)^{\ell-i} x_{n-i} y_{i} D_{1, r}(n-\ell+j ; y) .
$$

Considering all possible $j, \ell$ and $i$ gives the sum of the weights of all members of

$$
\mathcal{P}_{n, k, r}=\cup_{j=0}^{k} \cup_{\ell=0}^{n} \cup_{i=0}^{\ell} \mathcal{P}_{n, k, r}^{(j, \ell, i)}
$$


We now define an involution on $\mathcal{P}_{n, k, r}$ in two steps as follows. First suppose $\rho \in \mathcal{P}_{n, k, r}$ is such that the cycles of $\alpha$ within at least one of the blocks $B$ of $\delta$ contain two or more elements of $[k]$ altogether. We assume that the blocks of $\delta$ are arranged left-to-right in ascending order of smallest items contained within, where all free elements are considered less than all cycles and cycles are compared by comparing sizes of first elements. Suppose further that $B$ is leftmost when the blocks of $\delta$ are ordered as described. Within $B$, identify the smallest two elements of $[k]$ contained within cycles of $\alpha$, which we will denote by $u$ and $v$ where $u<v$. If $u$ and $v$ occur in the same cycle of $\alpha$ as $(u \ldots v \ldots)$, then we break the cycle at $v$ to obtain two cycles $(u \ldots)$, $(v \ldots)$, and if $u$ and $v$ occur in different cycles, then we reverse this operation. In the process, we keep the associations of any members of $\gamma$ the same, whence the weight is preserved as $m=1$. On the other hand, since the number of cycles of $\alpha$ changes by one and $|\gamma|=\ell-i$ stays the same, the sign is reversed.

Thus, we may assume $\rho \in \mathcal{P}_{n, k, r}$ is such that each block of $B$ contains either no cycles of $\alpha$ at all or a single cycle of $\alpha$ of length one (which implies $j=k$ and $\alpha$ is the identity permutation). In this case, to define an involution, we first identify the smallest block $C$ of $\delta$ (relative to the ordering described above) containing a one cycle $(x)$ of $\alpha$ such that (i) some element of $\gamma$ is associated with the element $x$, or (ii) some member of $\beta$ belongs to $C$ as a free element (possibly both (i) and (ii) occur). Let $w$ be the smallest element of $\gamma \cup \beta$ that either (I) is associated with $x$ or (II) lies in block $C$ as a free element. We switch options (I) and (II) with respect to $w$. Note that this operation preserves the $x$ - and the $y$-weights since $|\beta|+|\gamma|=(n-\ell)+(\ell-i)=n-i$ does not change. Moreover, since $m=1$, possibly adding a free element to a non-special block or removing it does not change the weight of $\delta$ either. Finally, the sign is reversed since $\alpha$ stays the same but $|\gamma|$ changes by one.

Combining the two mappings above then yields an involution of $\mathcal{P}_{n, k, r}$ whose set of survivors $\mathcal{P}_{n, k, r}^{*}$ all have positive sign since $j=k$ and $\gamma=\varnothing$. Furthermore, within each survivor, the cycles of $\alpha$ must all belong to distinct blocks of $\delta$ with no cycle sharing a block with a free element. Thus, a cycle $(z)$ of $\alpha$ either comprises its own block of $\delta$ or belongs to a block of the form $\left\{r^{\prime},(z)\right\}$, where $r^{\prime} \in[r]$. So to enumerate members of $\mathcal{P}_{n, k, r}^{*}$, let $i$ denote here the number of elements of $[r]$ containing a cycle of $\alpha$ within its block in $\delta$. There are $\left(\begin{array}{c}r \\ i\end{array}\right)\left(\begin{array}{l}k \\ i\end{array}\right) i$ ! ways in which to select the elements of $[r]$ and cycles of $\alpha$ and then to combine them in $i$ doubleton blocks. Each of the remaining $k-i$ cycles goes in its own block and thus they contribute weight $y^{k-i}$ altogether, while the elements of $\beta$ and the remaining $r-i$ special elements are combined according to a partition enumerated by $D_{1, r-i}(j ; y)$ where $j$ here denotes $|\beta|$. Considering all possible $i$ and $j$ then implies that $\left|\mathcal{P}_{n, k, r}^{*}\right|$ is given by the left-hand side of (13), which completes the proof.

Corollary 2.4.1. We have

$$
\begin{aligned}
\sum_{j=0}^{n}\left(\begin{array}{l}
n \\
j
\end{array}\right) a^{j} b^{n-j} \sum_{i=0}^{r}\left(\begin{array}{l}
r \\
i
\end{array}\right)\left(\begin{array}{l}
k \\
i
\end{array}\right) i ! y^{k-i} D_{1, r-i}(j ; y)=\sum_{j=0}^{k}(-1)^{k-j}\left[\begin{array}{l}
k \\
j
\end{array}\right] \sum_{\ell=0}^{n}\left(\begin{array}{l}
n \\
\ell
\end{array}\right) a^{\ell}(b-a k)^{n-\ell} \\
\times D_{1, r}(\ell+j ; y) .
\end{aligned}
$$


In particular,

$$
y^{k} \sum_{j=0}^{n}\left(\begin{array}{l}
n \\
j
\end{array}\right) a^{j} b^{n-j} B(j ; y)=\sum_{j=0}^{k}(-1)^{k-j}\left[\begin{array}{l}
k \\
j
\end{array}\right] \sum_{\ell=0}^{n}\left(\begin{array}{l}
n \\
\ell
\end{array}\right) a^{\ell}(b-a k)^{n-\ell} B(\ell+j ; y) .
$$

Proof. Taking $x_{j}=a^{j}$ and $y_{j}=b^{j}$ for all $j \geq 0$ in (13) gives

$$
\begin{aligned}
& \sum_{j=0}^{n}\left(\begin{array}{l}
n \\
j
\end{array}\right) a^{j} b^{n-j} \sum_{i=0}^{r}\left(\begin{array}{l}
r \\
i
\end{array}\right)\left(\begin{array}{l}
k \\
i
\end{array}\right) i ! y^{k-i} D_{1, r-i}(j ; y) \\
& =\sum_{j=0}^{k}(-1)^{k-j}\left[\begin{array}{l}
k \\
j
\end{array}\right] \sum_{\ell=0}^{n} \sum_{i=0}^{\ell}\left(\begin{array}{l}
n \\
\ell
\end{array}\right)\left(\begin{array}{l}
\ell \\
i
\end{array}\right)(-k)^{\ell-i} a^{n-i} b^{i} D_{1, r}(n-\ell+j ; y) \\
& =\sum_{j=0}^{k}(-1)^{k-j}\left[\begin{array}{l}
k \\
j
\end{array}\right] \sum_{\ell=0}^{n}\left(\begin{array}{l}
n \\
\ell
\end{array}\right) a^{n-\ell}(b-a k)^{\ell} D_{1, r}(n-\ell+j ; y),
\end{aligned}
$$

by the binomial theorem. Replacing $\ell$ by $n-\ell$ in the last equality yields (14). Letting $r=0$ in (14) gives (15).

Letting $y=1$ and $r=0$ in (13) yields the following Bell number formula.

\section{Corollary 2.4.2. We have}

$$
\sum_{j=0}^{n}\left(\begin{array}{l}
n \\
j
\end{array}\right) x_{j} y_{n-j} B(j)=\sum_{j=0}^{k}(-1)^{k-j}\left[\begin{array}{l}
k \\
j
\end{array}\right] \sum_{\ell=0}^{n} \sum_{i=0}^{\ell}\left(\begin{array}{l}
n \\
\ell
\end{array}\right)\left(\begin{array}{l}
\ell \\
i
\end{array}\right)(-k)^{\ell-i} x_{n-i} y_{i} B(n-\ell+j) .
$$

Remark: Either setting $y=1$ in (15) or $x_{j}=a^{j}$ and $y_{j}=b^{j}$ for all $j$ in (16) gives (4). In [5], it was shown algebraically that (16) holds in the particular cases when $x_{j}, y_{j}$ assume (independently) the values of one of the following five sequences of numbers: constant 1 , Fibonacci $F_{j}$, Lucas $L_{j}$, Bernoulli $\mathfrak{B}_{j}$ or Euler $E_{j}$. Formula (16) was also shown to hold when $x_{j}=(-1)^{j} F_{2 j}$ or $(-1)^{j} L_{2 j}$ and $y_{j}=1$ for all $j$.

We conclude this section with the following generalization of (5).

Theorem 2.5. If $n, k, r \geq 0$, then

$$
\begin{aligned}
\sum_{j=0}^{n+k}\left(\begin{array}{c}
n+k \\
j
\end{array}\right) x_{j} y_{n+k-j} D_{m, r}(j ; y)=\sum_{p=0}^{k} \sum_{\ell=0}^{k} \sum_{j=0}^{n} \sum_{i=0}^{j}\left(\begin{array}{c}
n \\
j
\end{array}\right)\left(\begin{array}{c}
k \\
\ell
\end{array}\right)\left(\begin{array}{l}
j \\
i
\end{array}\right) x_{n+\ell+i-j} y_{k+j-\ell-i} \\
\times(p m)^{n-j} y^{p} W_{m, r}(\ell, p) D_{m, r}(i ; y)
\end{aligned}
$$

Proof. Let $\mathcal{T}_{n, k, r}^{(t)}$ where $0 \leq t \leq n+k$ denote the set of ordered pairs $(\alpha, \beta)$, where $\beta \subseteq$ $[r+1, r+n+k]$ with $|\beta|=n+k-t$ and $\alpha$ is a partition of the elements of $([r+1, r+n+k]-\beta) \cup[r]$ according to a member of $\mathcal{B}_{m, r}(t)$. Let the weight of $(\alpha, \beta) \in \mathcal{T}_{n, k, r}^{(t)}$ be given by $x_{t} y_{n+k-t} \mathrm{wght}(\alpha)$, where wght $(\alpha)$ is the weight assigned to $\alpha$ when considered as a member of $\mathcal{B}_{m, r}(t)$ and counted in the distribution polynomial $D_{m, r}(t ; y)$. Then the left side of (17) is seen to give the sum of the weights of all members of $\mathcal{T}_{n, k, r}=\cup_{t=0}^{n+k} \mathcal{T}_{n, k, r}^{(t)}$. 
We compute this sum of weights of the $(\alpha, \beta) \in \mathcal{T}_{n, k, r}$ now in a different way. First suppose that exactly $p$ of the non-special blocks of the partition $\alpha$ contain at least one member of the set $I=[r+1, r+k]$, where $0 \leq p \leq k$. We then select $\ell$ elements of $I$ and form a partition together with the elements of $[r]$ according to a member of $\mathcal{B}_{m, r}(\ell, p)$, which can be effected in $W_{m, r}(\ell, p)$ ways. The remaining $k-\ell$ members of $I$ then go in $\beta$. Now suppose exactly $n-j$ elements of the set $J=[r+k+1, r+k+n]$ belong to a non-special block of $\alpha$ containing at least one member of $I$. Then there are $\left(\begin{array}{l}n \\ j\end{array}\right)(\mathrm{pm})^{n-j}$ ways in which to select and insert these elements into one of the $p$ non-special blocks already present (note that these added elements are each colored in one of $m$ ways since they are non-minimal). Then select $i$ of the unchosen $j$ members of $J$ to go in new non-special blocks of $\alpha$ or in the special blocks, which can be effected in $\left(\begin{array}{l}j \\ i\end{array}\right) D_{m, r}(i ; y)$ ways. The remaining $j-i$ members of $J$ are understood to go in $\beta$. Thus, we have $|\alpha|=\ell+n-j+i$ and $|\beta|=k-\ell+j-i$, which become the respective subscripts of the $x_{j}$ - and $y_{j}$-sequence factors appearing in the weight. Finally, one must multiply by $y^{p}$ to account for the $p$ non-special blocks of $\alpha$ above containing at least one member of $I$. Considering all possible $p, \ell, j$ and $i$ implies that the sum of the weights of all members of $\mathcal{T}_{n, k, r}$ is also given by the right side of (17), which completes the proof.

Remark. Allowing the subset of $J$ enumerated by $n-j$ in the preceding proof to include also those members of $J$ belonging to special blocks yields a variant of (17) wherein $(p m)^{n-j} D_{m, r}(i ; y)$ is replaced by $(r+p m)^{n-j} D_{m, 0}(i ; y)$ on the right side of (17), with all other factors the same as before. Note that our argument for (17) is seen to extend the proof given by Spivey [14] of formula (7) above, which corresponds to the case when $x_{j}=y=m=1, y_{j}=\delta_{j, 0}$ and $r=0$.

Setting $m=y=1$ and $r=0$ in (17) yields the following Bell number identity.

Corollary 2.5.1. We have

$$
\sum_{j=0}^{n+k}\left(\begin{array}{c}
n+k \\
j
\end{array}\right) x_{j} y_{n+k-j} B(j)=\sum_{p=0}^{k} \sum_{\ell=0}^{k} \sum_{j=0}^{n} \sum_{i=0}^{j}\left(\begin{array}{l}
n \\
j
\end{array}\right)\left(\begin{array}{l}
k \\
\ell
\end{array}\right)\left(\begin{array}{l}
j \\
i
\end{array}\right) x_{n+\ell+i-j} y_{k+j-\ell-i} p^{n-j}\left\{\begin{array}{l}
\ell \\
p
\end{array}\right\} B(i) .
$$

Remark: Formula (18) reduces to (5) when $x_{j}=a^{j}$ and $y_{j}=b^{j}$. It was also shown in [5] that (18) holds in particular when $x_{j}$ and $y_{j}$ coincide with one of the five number sequences mentioned in the first remark above as well as when $x_{j}=(-1)^{j} F_{2 j}$ or $(-1)^{j} L_{2 j}$ and $y_{j}=1$.

\section{A further extension of (5)}

Let $\mathcal{L}(n, k)$ denote the set of partitions of $[n]$ into $k$ blocks where elements within a block may be written in any order. Then $|\mathcal{L}(n, k)|$ is given by the Lah number $\frac{n !}{k !}\left(\begin{array}{l}n-1 \\ k-1\end{array}\right)$ if $n, k \geq 1$ (see, e.g., [13, A008297]), with $\mathcal{L}(n)=\cup_{k=0}^{n} \mathcal{L}(n, k)$ having cardinality given by [13, A000262]. We now recall the generalized Stirling polynomials $\mathcal{G}_{s, t}(n, k)$ that were studied in [7] which arise as the joint distribution for a pair of statistics on $\mathcal{L}(n, k)$. They are defined recursively by

$$
\mathcal{G}_{s, t}(n, k)=\mathcal{G}_{s, t}(n-1, k-1)+(s(n-1)+t k) \mathcal{G}_{s, t}(n-1, k), \quad n, k \geq 1,
$$


with $\mathcal{G}_{s, t}(n, 0)=\delta_{n, 0}$ and $\mathcal{G}_{s, t}(0, k)=\delta_{k, 0}$ for $n, k \geq 0$. Note that $\mathcal{G}_{1,0}(n, k)=\left[\begin{array}{l}n \\ k\end{array}\right]$ and $\mathcal{G}_{0,1}(n, k)=\left\{\begin{array}{l}n \\ k\end{array}\right\}$.

We now describe a combinatorial interpretation for $\mathcal{G}_{s, t}(n, k)$. Within a block $B$ of $\lambda \in \mathcal{L}(n)$ and $i \in B$, we will say that $i$ is a record low of $\lambda$ if there are no elements $j<i$ to the left of $i$ within $B$. If $\lambda \in \mathcal{L}(n)$, then let $\operatorname{rec}^{*}(\lambda)$ denote the total number of record lows of $\lambda$ (within all of its blocks) which do not correspond to a minimal element of some block. Let nrec $(\lambda)$ denote the number of elements of $[n]$ which are not record lows of $\lambda$. Comparing recurrences and initial values, one can show that $\mathcal{G}_{s, t}(n, k)$ is the joint distribution for the nrec and rec* statistics on $\mathcal{L}(n, k)$, i.e.,

$$
\mathcal{G}_{s, t}(n, k)=\sum_{\lambda \in \mathcal{L}(n, k)} s^{\mathrm{nrec}(\lambda)} t^{\mathrm{rec}(\lambda)}, \quad n, k \geq 1
$$

see [7, Theorem 5.1].

Let $\mathcal{G}_{s, t}(n)=\sum_{k=0}^{n} \mathcal{G}_{s, t}(n, k)$ for $n \geq 0$. If $a$ and $b$ are variables and $i$ is a positive integer, then let $[a: b]_{i}=a(a+b) \cdots(a+(i-1) b)$, with $[a: b]_{0}=1$. We have the following extension of (5) involving generalized Stirling polynomials.

Theorem 3.1. If $n, k \geq 0$, then

$$
\begin{aligned}
\sum_{j=0}^{n+k}\left(\begin{array}{c}
n+k \\
j
\end{array}\right) x_{j} y_{n+k-j} \mathcal{G}_{s, t}(j)=\sum_{p=0}^{k} \sum_{\ell=0}^{k} \sum_{j=0}^{n} \sum_{i=0}^{j}\left(\begin{array}{l}
n \\
j
\end{array}\right)\left(\begin{array}{l}
k \\
\ell
\end{array}\right)\left(\begin{array}{l}
j \\
i
\end{array}\right) x_{n+\ell+i-j} y_{k+j-\ell-i} \\
\times[p t+\ell s: s]_{n-j} \mathcal{G}_{s, t}(\ell, p) \mathcal{G}_{s, t}(i) .
\end{aligned}
$$

Proof. We proceed in a manner similar to the proof of Theorem 2.5 above. Consider ordered pairs $(\alpha, \beta)$, where $\beta \subseteq[n+k]$ with $|\beta|=n+k-m$ for some $0 \leq m \leq n+k$ and $\alpha$ is a Lah distribution on the elements of $[n+k]-\beta$. We define the weight of $(\alpha, \beta)$ as $x_{m} y_{n+k-m}$ wght $(\alpha)$, where $\operatorname{wght}(\alpha)=s^{\text {nrec }(\alpha)} t^{\text {rec* }(\alpha)}$. Then, by (20), the left side of (21) gives the sum of the weights of all possible ordered pairs $(\alpha, \beta)$. The right side of (21) also achieves this upon proceeding as in the proof of (17) above (in the case $r=0$ ) and making the appropriate modifications. Note that there are now $[p t+\ell s: s]_{n-j}$ ways in which to add the $n-j$ elements chosen from $J$ that are to go in blocks containing at least one member of $I$, as there are $p t+\ell s$ possibilities for the position of the smallest chosen element, $p t+(\ell+1) s$ possibilities for the position of the second smallest and so on. Equating the two expressions for the sum of the weights of the $(\alpha, \beta)$ implies (21).

Note that (21) reduces to (5) when $x_{j}=a^{j}, y_{j}=b^{j}, s=0$ and $t=1$. Taking $s=1$ and $t=0$ instead gives

$$
\sum_{j=0}^{n+k}\left(\begin{array}{c}
n+k \\
j
\end{array}\right) a^{j} b^{n+k-j} j !=\sum_{p=0}^{k} \sum_{\ell=0}^{k} \sum_{j=0}^{n} \sum_{i=0}^{j}\left(\begin{array}{l}
n \\
j
\end{array}\right)\left(\begin{array}{l}
k \\
\ell
\end{array}\right)\left(\begin{array}{l}
j \\
i
\end{array}\right) a^{n+\ell+i-j} b^{k+j-\ell-i} \ell^{n-j}\left[\begin{array}{l}
\ell \\
p
\end{array}\right]
$$

where $\ell^{\bar{j}}=\ell(\ell+1) \cdots(\ell+j-1)$, which is an analogue of $(5)$ for permutations. A similar formula involving the Lah numbers is obtained if one sets $s=t=1$. Taking $s=1, t=0, x_{j}=1$ and $y_{j}=\delta_{j, 0}$ in (21) gives 


$$
(n+k) !=\sum_{p=0}^{k} \sum_{j=0}^{n}\left(\begin{array}{l}
n \\
j
\end{array}\right) k^{\overline{n-j}}\left[\begin{array}{l}
k \\
p
\end{array}\right] j !,
$$

which is an analogue of (7) for permutations, see [9, Theorem 1]. Taking $s=t=1$ instead yields a comparable formula for the Lah numbers.

\section{References}

[1] Benoumhani, M. (1997). On some numbers related to Whitney numbers of Dowling lattices, Adv. in Appl. Math., 19, 106-116.

[2] Cheon, G.-S. \& Jung, J.-H. (2012). r-Whitney numbers of Dowling lattices, Discrete Math., 312, 2337-2348.

[3] Dowling, T. A. (1973). A class of geometric lattices based on finite groups, J. Combin. Theory Ser. B, 14, 61-86. (Erratum: (1973). J. Combin. Theory Ser. B, 15, 211.)

[4] Gould, H. W. \& Quaintance, J. (2008). Implications of Spivey's Bell number formula, J. Integer Seq., 11, Art. 08.3.7.

[5] Komatsu, T. \& Pita-Ruiz, C. (2018). Some formulas for Bell numbers, Filomat, 32:11, 3881-3889.

[6] Mansour, T., Ramírez, J. L. \& Shattuck, M. (2017). A generalization of the $r$-Whitney numbers of the second kind, J. Comb., 8:1, 29-55.

[7] Mansour, T., Schork, M. \& Shattuck, M. (2011). On a new family of generalized Stirling and Bell numbers, Electron. J. Combin., 18, \#P77.

[8] Mező, I. (2010). A new formula for the Bernoulli polynomials, Results Math., 58(3), 329335.

[9] Mező, I. (2012). The dual of Spivey's Bell number formula, J. Integer Seq., 15, Art. 12.2.4.

[10] Mihoubi, M. \& Belbachir, H. (2014). Linear recurrences for $r$-Bell polynomials, J. Integer Seq., 17, Art. 14.10.6.

[11] Rahmani, M. (2014). Some results on Whitney numbers of Dowling lattices, Arab J. Math. Sci., 20(1), 11-27.

[12] Shattuck, M. (2016). Generalizations of Bell number formulas of Spivey and Mezó, Filomat, 30:10, 2683-2694.

[13] Sloane, N. J. A. (2010). On-line Encyclopedia of Integer Sequences, Available online at http://oeis.org.

[14] Spivey, M. Z. (2008). A generalized recurrence for Bell numbers, J. Integer Seq., 11, Art. 08.2.5.

[15] Xu, A. (2012). Extensions of Spivey's Bell number formula, Electron. J. Combin., 19(2), \#P6. 